\title{
Numerical Simulation of LVAD Inflow Cannulas with Different Tip
}

\author{
Guang-Mao Liu, ${ }^{1}$ Hai-Bo Chen, ${ }^{1}$ Fu-Liang Luo, ${ }^{2}$ Yan Zhang, ${ }^{2}$ Han-Song Sun, ${ }^{2}$ \\ Jian-Ye Zhou, ${ }^{1}$ and Sheng-Shou $\mathrm{Hu}^{1}$ \\ ${ }^{1}$ Key Laboratory of Cardiovascular Regenerative Medicine, Ministry of Health, State Key Laboratory of Cardiovascular Disease, \\ Fuwai Hospital, National Center for Cardiovascular Diseases, Chinese Academy of Medical Sciences and Peking Union \\ Medical College, Beijing 100037, China \\ ${ }^{2}$ Cardiovascular Surgery, State Key Laboratory of Cardiovascular Disease, Fuwai Hospital, National Center for Cardiovascular Diseases, \\ Chinese Academy of Medical Sciences and Peking Union Medical College, Beijing 100037, China
}

Correspondence should be addressed to Jian-Ye Zhou, jianyezhou@yahoo.com

Received 23 December 2011; Accepted 29 February 2012

Academic Editor: Mandar Tabib

Copyright (C) 2012 Guang-Mao Liu et al. This is an open access article distributed under the Creative Commons Attribution License, which permits unrestricted use, distribution, and reproduction in any medium, provided the original work is properly cited.

\begin{abstract}
The tip structure of LVAD inflow cannula is one of major factors to lead adverse events such as thrombosis and suction leading to obstruction. In this research, four kinds of tips that had been used in inflow cannulas were selected and designed. The flow field of the four inflow cannulas inserted into the apex of left ventricle (LV) was numerically computed by computational fluid dynamics. The flow behavior was analyzed in order to compare the blood compatibility and suction in left ventricle and cannulas after the inflow cannulas with different tips were inserted to the apex of LV. The results showed that the cannula tip structure affected the LVAD performance. Among these four cannulas, the trumpet-tipped inflow cannula owned the best performance in smooth flow velocity distribution without backflow or low-velocity flow so that it was the best in blood compatibility. Nevertheless, the caged tipped cannula was the worst in blood compatibility. And the blunt-tipped and beveled tipped inflow cannulas may obstruct more easily than trumpet and caged tipped inflow cannulas because of their shape. The study indicated that the trumpet tip was the most preferable for the inflow cannula of long-term LVAD.
\end{abstract}

\section{Introduction}

LVADs have been widely applied to assist the circulation of heart failure (HF) patients. Some LVADs can prolong HF patients' lives for more than two years [1-5]. Now, most LVADs are equipped with complete accessories such as designed inflow and outflow cannulas to ensure that the LVADs can work safely and longer. The inflow cannula is the connection between left ventricle and LVAD. The inflow cannulas are mainly made of good blood compatibility materials such as titanium alloy, silicon rubber, or polythene. The shapes of inflow cannulas include blunt, beveled, caged, trumpet, and beak [6]. For example, the inflow cannula of Berlin Heart INCOR is made of silicon rubber with beakshaped tip $[7,8]$. And the inflow cannula of Debakey LVAD is made of titanium alloy with trumpet shaped tip $[9,10]$.
The tip shape is a critical factor to fluid dynamic performance. The flow field and stress distribution in left ventricle and cannulas depend on the inflow cannulas tip structure at a certain flow rate and operative procedures such as insertion depth and insertion angle. Different tip structures of inflow cannulas can cause various degree of thrombosis and suction leading to obstruction that may further influence the LVAD's service life and the survival of the patients [11, 12]. Thrombosis is mainly caused by static and/or vortical flow in the inflow cannulas.

Mechanical hemolysis is another important aspect of the inflow cannulas. The hemolysis in the inflow cannulas is mainly caused by the high shear stress of flow in the inflow cannulas. The effective numerical prediction of mechanical hemolysis has been built [13-15]. In vitro and in vivo mechanical hemolysis tests have been studied previously [16-18]. 


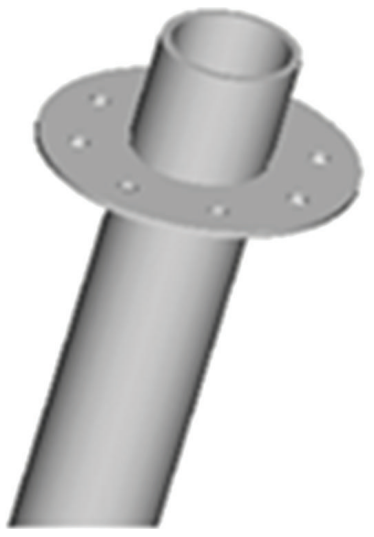

(a)

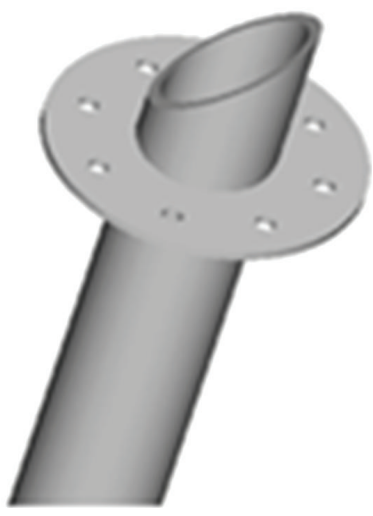

(b)

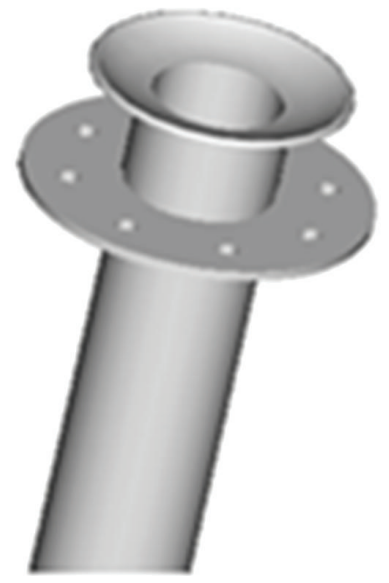

(c)

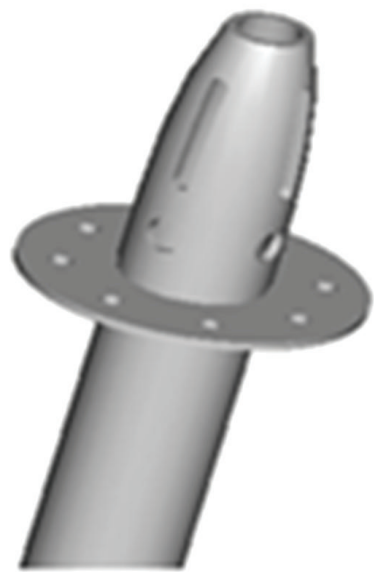

(d)

FIGURE 1: 3D model of inflow cannula tip: (a) blunt, (b) beveled, (c) trumpet, and (d) caged.

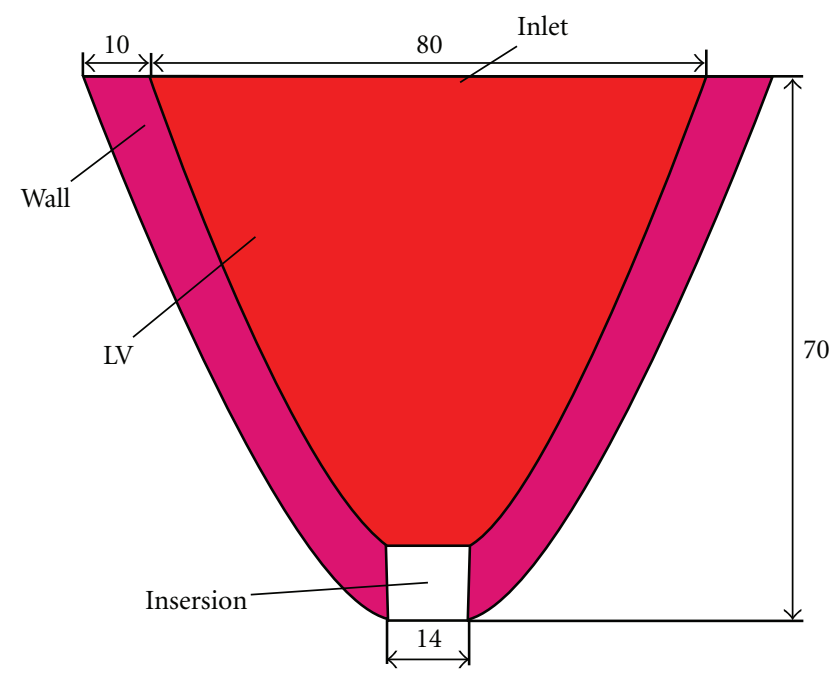

FIGURE 2: LV geometry model used to compare the inflow cannula tip.

To obtain a good performance in blood compatibility, the tip shape is the crucial factor to reduce static and vortical flow area in an inflow cannula design. The objective of this paper is to find which kind of tip shape is better for LVAD inflow cannula. The fluid dynamics of the inflow cannula are studied with numerical simulation or test in a mock loop model. Numerical simulation can simulate and analyze the flow velocity, stress distribution, and wall shear stress of cannula insertion $[19,20]$. In this paper, we built the numerical model of blunt, beveled, caged, and trumpet-tipped cannulas inserted into left ventricle, respectively. The velocity distribution and stress distribution around the cannula tips and in cannulas were calculated. The blood compatibility and risk of suction leading to obstruction [21] of the four insertions were compared. The analysis results can help us select tip shape and design an inflow cannula which is suitable for long-term LVAD.

\section{Materials and Methods}

2.1. Geometry Model. Figure 1 illustrated the 3D model of four kinds of inflow cannulas with different tip shapes built by Pro-E software. The cannulas were made of titanium alloy. The four cannulas were assumed to be in the same size where the inner diameter was $d=12 \mathrm{~mm}$, and the wall thickness was $s=1 \mathrm{~mm}$. And they were considered as straight pipes to eliminate effects of curved portion. The cannulas' length out of ventricle is $l=46 \mathrm{~mm}$.

Figure 2 showed the geometry model of LV for calculation. According to Fraser et al. and Tsukiya et al. $[19,22]$, the geometry of the left ventricle was simplified without regarding the heart real geometry, insertion depth, and insertion angle. The insertion point was assumed the apex of left ventricle. The inlet surface diameter of LV was set to $80 \mathrm{~mm}$. The LV wall thickness was $10 \mathrm{~mm}$ and uniform. The depth from the inlet surface to the apex was $70 \mathrm{~mm}$.

2.2. Computational Model. Four computational models of cannula inserted into LV were built by computational fluid dynamics software Workbench (ANSYS, Inc, Canonsburg, Pennsylvania, United States) according to the geometry models in Figures 1 and 2. Figure 3 demonstrated the typical $\backslash$ linebreak computational grid for the four inflow cannulas. We adopted unstructured numeric grid. Furthermore, blood was assumed to be Newtonian with density of $\rho=$ $1059 \mathrm{~kg} / \mathrm{m}^{3}$ and viscosity of $\mu=0.0035 \mathrm{~kg} / \mathrm{m} \cdot \mathrm{s}$. The average flow rate was set to $Q=5 \mathrm{~L} / \mathrm{min}$. Thus the inlet mass flow was $0.0088 \mathrm{~kg} / \mathrm{s}$. The static outlet pressure was set to $0 \mathrm{Mpa}$. The LV wall and cannula wall were considered to be stiffness boundary and immobile. The fluid-structure interaction simulation was complicated, so the interaction was not involved in this study $[19,20,22]$.

The Reynolds number was used as the index to estimate the flow to be laminar or turbulent. When the index is greater 


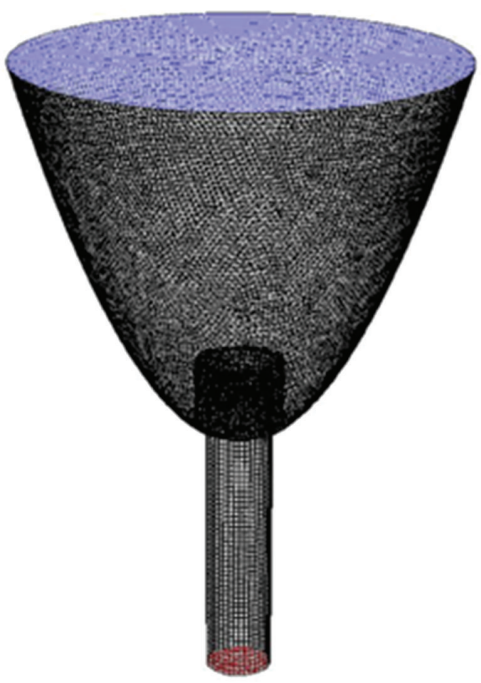

(a)

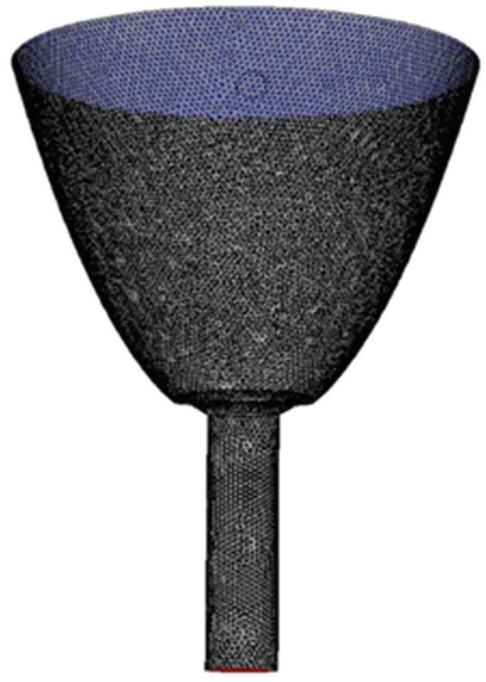

(c)

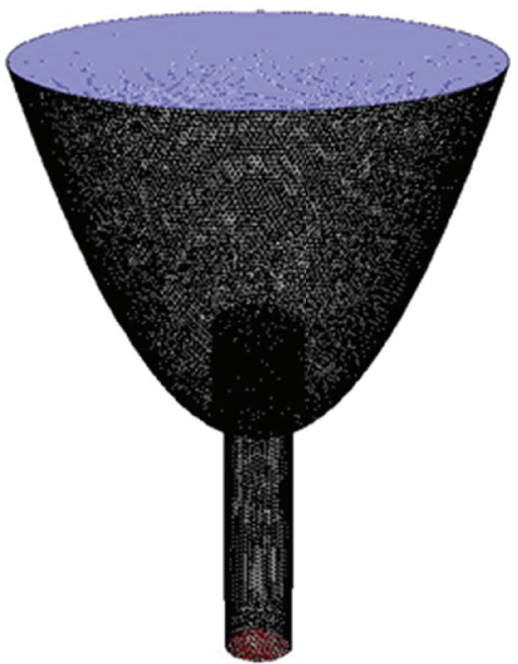

(b)

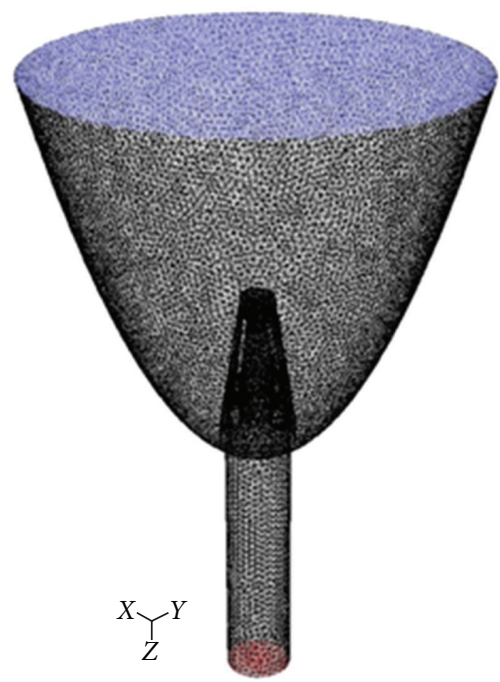

(d)

Figure 3: Numerical models of inflow cannulas insertion: (a) blunt, (b) beveled, (c) trumpet, and (d) caged.

than the critical Reynolds number, the flow is regarded as turbulent. The Reynolds number definition is

$$
\operatorname{Re}=\frac{V L}{v},
$$

where $V$ is the mean velocity of the section $(\mathrm{m} / \mathrm{s}), L$ is the characteristic length $(\mathrm{m})$, and $v$ is fluid dynamic viscosity $\left(\mathrm{m}^{2} / \mathrm{s}\right)$. For circular tube flow, the circular tube diameter $d$ is the characteristic length $L$. The Reynolds number of inflow cannula blood flow is

$$
\begin{aligned}
\operatorname{Re} & =\frac{V L}{\nu}=\frac{Q /\left(60 * 1000 \cdot \pi(d / 2000)^{2}\right) \cdot d / 1000}{\mu / \rho} \\
& =\frac{Q \rho}{15 \pi \mu d}=2658 .
\end{aligned}
$$

It is generally acknowledged that the critical Reynolds number of tube flow is 2320 [23]. So the flow in inflow cannula was turbulent flow. Therefore, $K-\varepsilon$ standard turbulence model was employed in numerical computation. The computational grid was generated by Workbench Fluent software. The unstructured numeric grid was generated from cannula surface.

\section{Results}

3.1. Velocity Distribution. Figure 4 showed velocity contour from inflow cannulas tip to outlet of four insertions. The flow velocity altered around the cannula tips and in cannulas. The flow velocity in ventricle was well distributed in the model of blunt and trumpet-tipped cannula. The maximal flow velocity was lower than $1 \mathrm{~m} / \mathrm{s}$. The velocity distribution was uniform and symmetrical along the $z$-axis after the blood flowed 


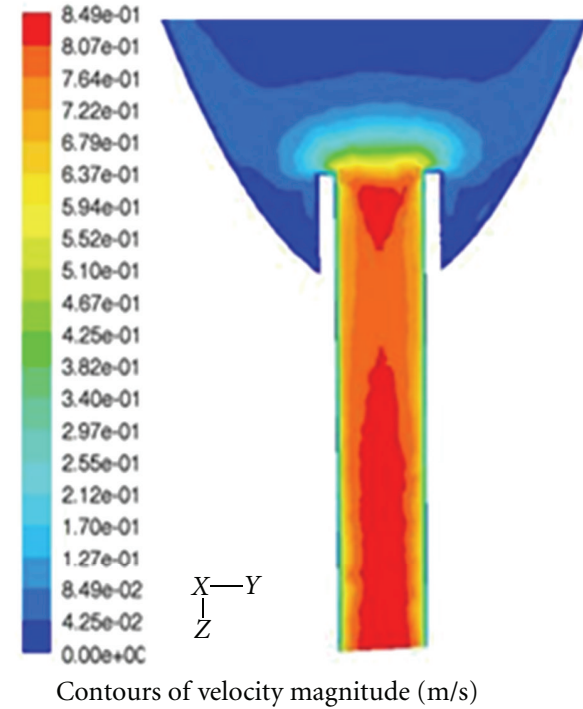

(a)

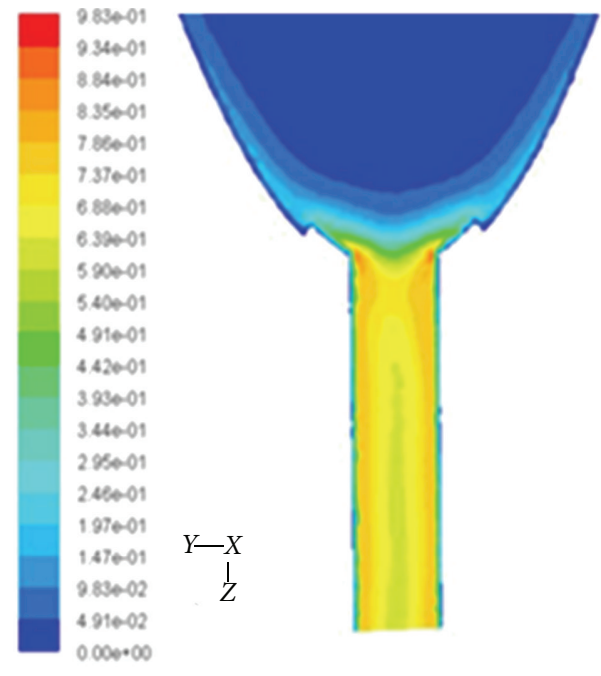

Contours of velocity magnitude $(\mathrm{m} / \mathrm{s})$

(c)

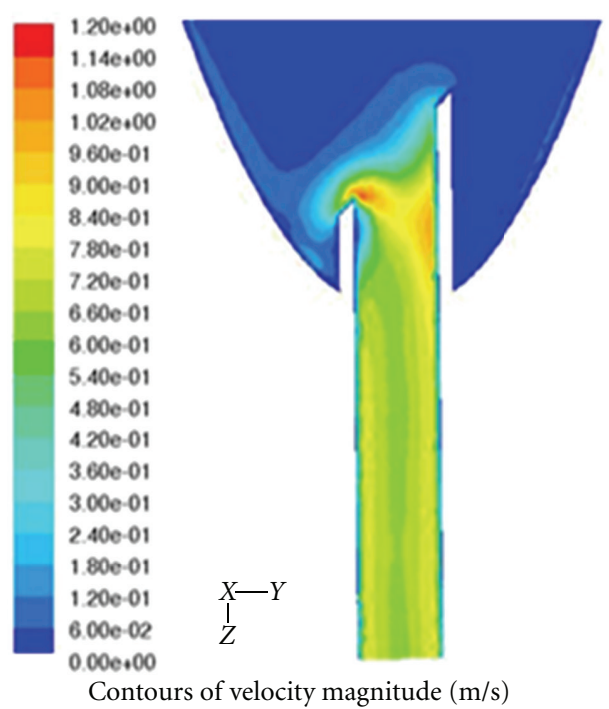

(b)

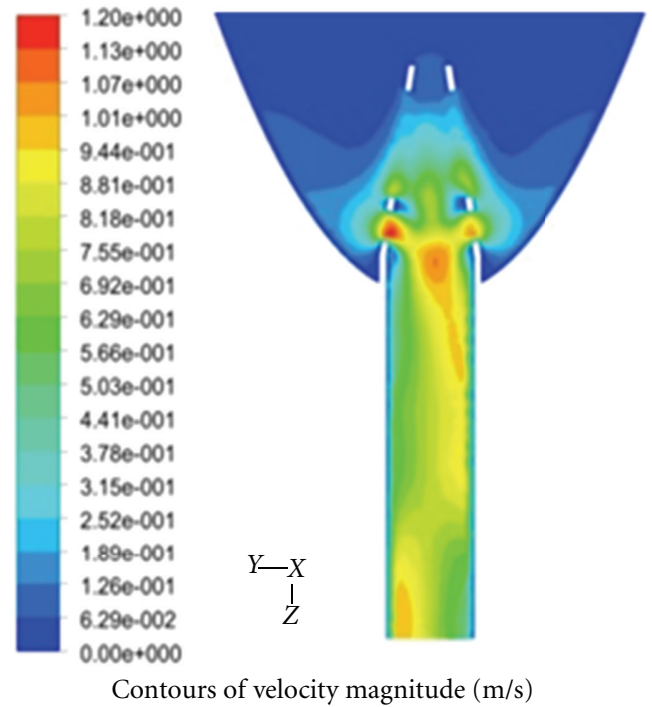

(d)

FIGURE 4: Velocity contour from tip to outlet: (a) blunt, (b) beveled, (c) trumpet, and (d) caged.

into the cannulas (Figures $4(\mathrm{a})$ and $4(\mathrm{c})$ ). The uniform distribution velocity in cannulas could improve the flow field in LVADs because the blood flows into LVAD from inflow cannulas directly. That meant the blood compatibility of LVADs was advanced. The beveled and caged tips disturbed the flow in left ventricle. The maximal flow velocity reached to $1.2 \mathrm{~m} / \mathrm{s}$. The velocity distribution was not even or symmetrical along the $z$-axis in the cannulas flow (Figures 4 (b) and $4(\mathrm{~d}))$. The uneven velocity distribution in these two cannulas adversely effected flow in LVAD.

Figure 5 showed velocity vectors around inflow cannulas tips. There was obvious vortex region between the blunttipped cannula outer wall and ventricular inner wall near the insertion point (Figure 5 (a)). The flow velocity in the region was slower than $0.1 \mathrm{~m} / \mathrm{s}$. So this area could be potential of thrombosis. Nevertheless, the area in blunt-tipped cannula was not likely to form thrombus because the flow in it was smooth. There were six obvious vortex regions near the caged tip (Figure 5(b)). Four of the six vortex regions were in caged tipped cannula and the minimum velocity was lower than $0.07 \mathrm{~m} / \mathrm{s}$. Additionally, turbulent flow was obvious near the inlet holes areas. Therefore these regions were likely to form clot. Figure 5(c) showed the velocity vector of beveled tipped inflow cannula. There were two vortex regions near insertion. One region was in left ventricle, and the other was in beveled tipped cannula. Both of these two regions were likely to form clot because the velocity was lower than $0.08 \mathrm{~m} / \mathrm{s}$. There was not vortex or flow stasis field in the trumpet tipped cannula (Figure 5(d)). The even velocity was $0.7 \mathrm{~m} / \mathrm{s}$ and well distributed. Thus trumpet-tipped cannula had good blood compatibility and was not likely to form thrombosis. 


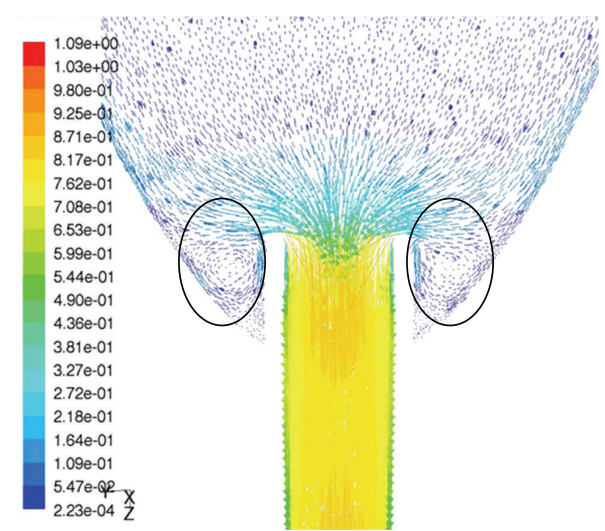

Velocity vectors colored by velocity magnitude $(\mathrm{m} / \mathrm{s})$

(a)

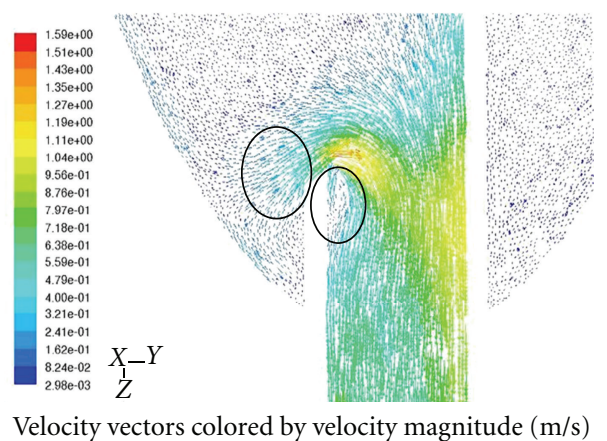

(c)

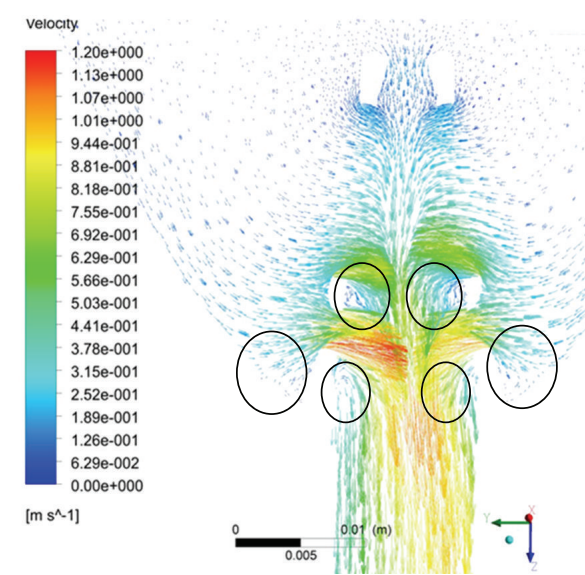

(b)

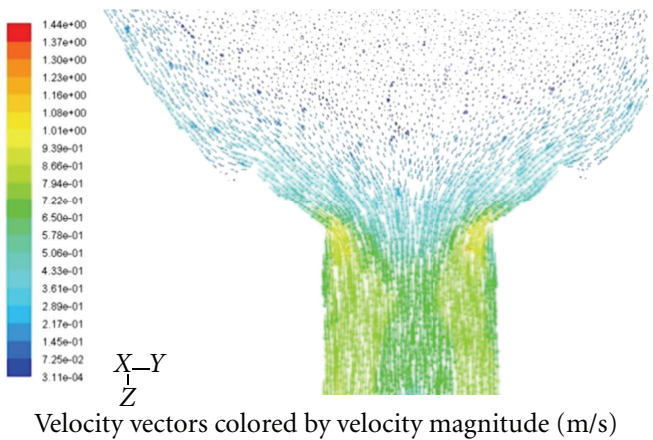

(d)

FIGURE 5: Velocity vector near tip: (a) blunt, (b) caged, (c) beveled, and (d) trumpet.

3.2. Static Pressure Distribution. Ventricular collapse and suction may occur when negative or low-pressure fields appeared. Furthermore, obstruction may happen if the tip could not resist the suction. Figure 6 showed static pressure distribution of four inflow cannulas insertion. The blunt tipped cannula did not create low-pressure field near the ventricular inner wall (Figure 6(a)). The beveled tipped cannula created one low-pressure field near the inflow bevel which was likely to ventricular collapsing (Figure 6(b)). The trumpet-tipped cannula had uniform low-pressure distribution around inlet (Figure 6(c)) which may cause ventricular collapse. Nevertheless, obstruction may not happen because the trumpet structure can resist suction. The caged tipped cannula created serious low-pressure field near inlet holes (Figure 6(d)). Ventricular collapse and suction were likely to occur. But the inlet could not be obstructed completely because not all the nine inlet holes could be obstructed at the same time.

3.3. Wall Shear Stress Distribution. Shear stress distribution was analyzed to evaluate hemolytic potential. Hemolysis is related to wall shear stress (WSS) value and blood exposure time [13-15, 24-26]. The computational fluid dynamics model with Eulerian formulation to estimate hemolysis in prosthesis device had been studied by Lacasse et al. [13], Song et al. [14], and Arvand et al. [15].
We estimated the damage to red cells with (3) [14]:

$$
\begin{aligned}
D & =\int_{\text {outlet }}^{\text {inlet }} 1.8 \times 10^{-6} \cdot \tau^{1.991} \cdot d T^{0.765} \\
& =\sum_{\text {outlet }}^{\text {inlet }} 1.8 \times 10^{-6} \cdot \tau^{1.991} \cdot \Delta T^{0.765},
\end{aligned}
$$

where $D$ symbolizes the blood damage index, $\tau$ is shear stress $(\mathrm{Pa})$, and $T$ is blood exposure time (s). The particle displacement is calculated using forward Euler integration of the particle velocity over time step $(\delta t)$ as $(4)$ :

$$
x_{i}^{n}=x_{i}^{o}+\frac{d x_{i}^{o}}{d t} \delta t
$$

where $x$ denotes the displacement and the superscripts $o$ and $n$ refer to old and new values, respectively.

The blood damage index of the four inflow cannulas was shown in Figure 7. The estimation value of blood damage was between $5 \times 10^{-5} \%$ to $20 \times 10^{-5} \%$. The acceptable estimation value of blood damage for LVAD was $10^{-3} \%$ [27, $28]$. So the blood damage in inflow cannula was negligible. The shear stress in inflow cannula was not able to cause hemolysis.

3.4. Limitations. This study eliminated the factors of real ventricle geometry, insertion depth, and insertion angle. But 


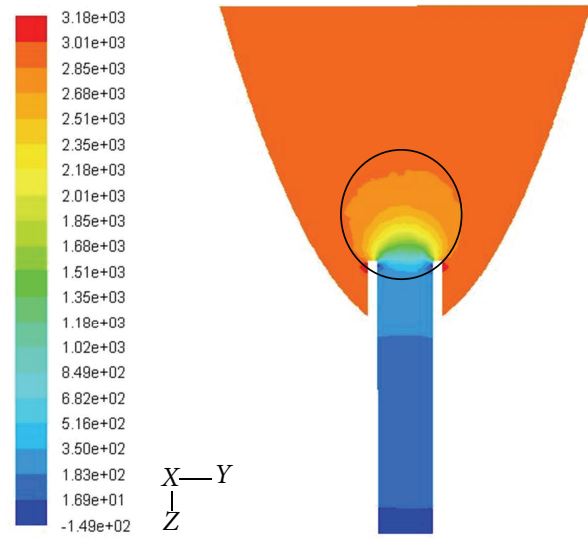

Contours of static pressure (pascal)

(a)

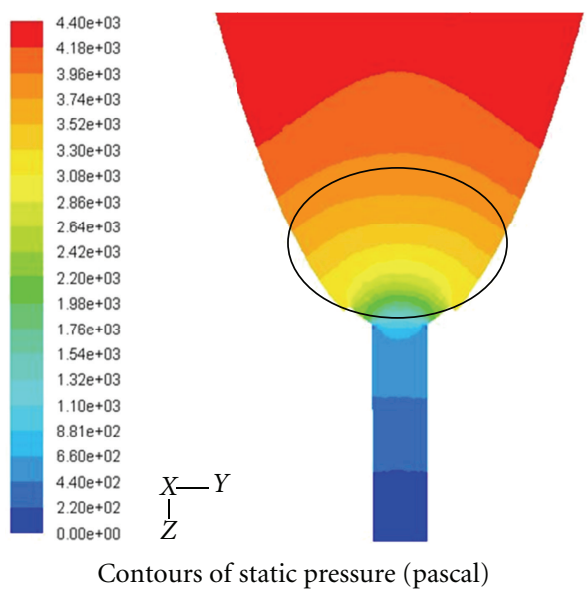

(c)

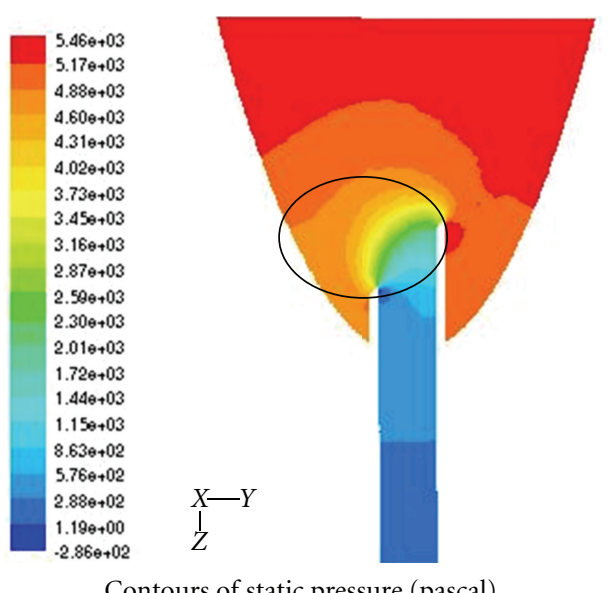

Contours of static pressure (pascal)

(b)
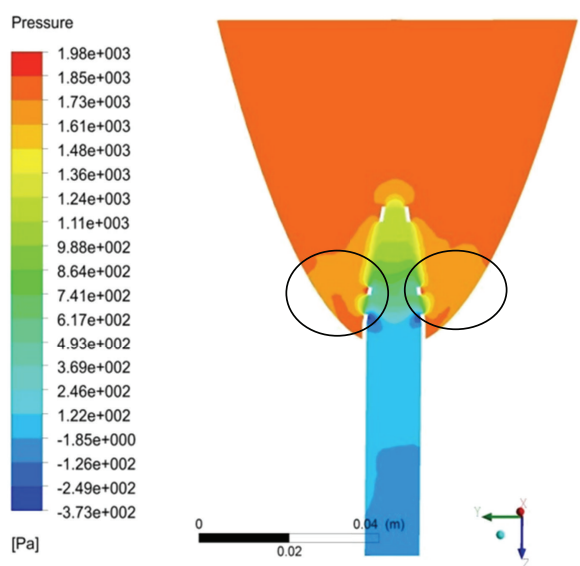

(d)

Figure 6: Contour of static pressure: (a) blunt, (b) beveled, (c) trumpet, and (d) caged.

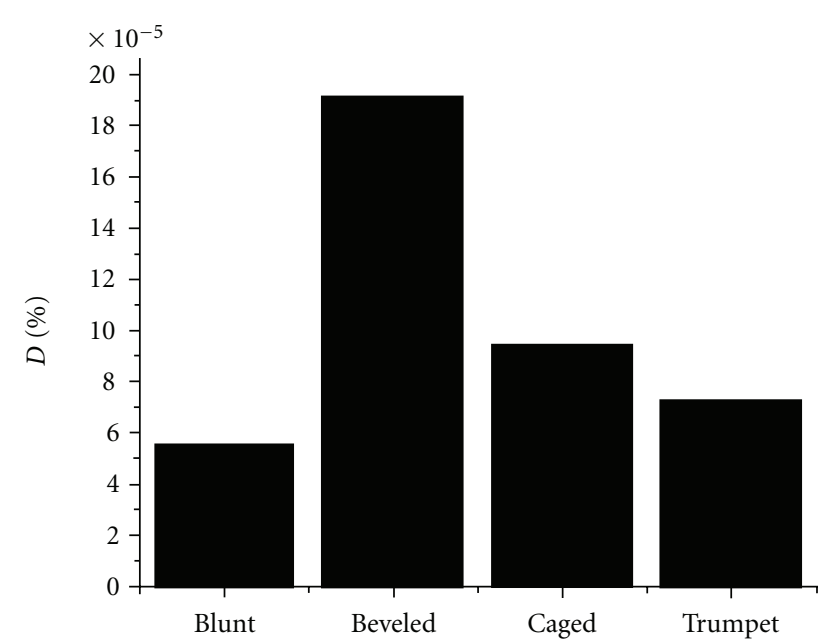

Figure 7: Blood damage index of inflow cannula.

actually, these factors may change the flow distribution in LV and inflow cannula and eventually cause the suction, and obstruction. So the effect of insertion depth and angle on thrombosis potential, suction and obstruction should be further studied in the future.

The other limitation in this study is the simplified boundary conditions with rigid cavity and uniform flow inlet assumption. In reality, the flow in LV is much more complicated. The flow and pressure in ventricle change all the time with the beating. As the purpose of this study is to compare the tip shapes of the inflow cannulas, we think that the simplified boundary conditions are acceptable. Of course, the final outcome should be verified in animal test. And based on the results, profound study with more exact conditions is need in the future.

\section{Conclusions}

Despite the limitation, trumpet tip structure had the best effect on blood compatibility and was hard to generate suction. So trumpet-tipped inflow cannula was the best inflow cannula for LVAD. The caged tip structure had the worst impact on blood compatibility. The thrombosis potential of caged tipped cannula for long-term use was substantial. 


\section{Acknowledgments}

This paper was supported by grants from National Hightech R\&D Program of China (863 Program) (no. 2006AA02Z4D3) and from state-level public service research institutes fund of basic research (2010-F08). The authors appreciated Mr. Lian-Hua Ma, from College of Mechanical Engineering and Applied Electronics Technology of Beijing University of Technology, for his great help in numerical calculation.

\section{References}

[1] E. A. Rose, A. C. Gelijns, A. J. Moskowitz et al., "Long-term use of a left ventricular assist device for end-stage heart failure," The New England Journal of Medicine, vol. 345, no. 20, pp. 1435-1443, 2001.

[2] M. C. Deng, L. B. Edwards, M. I. Hertz et al., "Mechanical circulatory support device database of the International Society for Heart and Lung Transplantation: third annual report2005," Journal of Heart and Lung Transplantation, vol. 24, no. 9, pp. 1182-1187, 2005.

[3] J. A. Morgan, R. John, V. Rao et al., "Bridging to transplant with the HeartMate left ventricular assist device: the Columbia Presbyterian 12-year experience," Journal of Thoracic and Cardiovascular Surgery, vol. 127, no. 5, pp. 1309-1316, 2004.

[4] S. Salzberg, M. Lachat, G. Zünd et al., "Left ventricular assist device as bridge to heart transplantation-lessons learned with the MicroMed DeBakey axial blood flow pump," European Journal of Cardio-thoracic Surgery, vol. 24, no. 1, pp. 113118, 2003.

[5] G. P. Noon, D. Morley, S. Irwin, and R. Benkowski, "Development and clinical application of the MicroMed DeBakey VAD," Current Opinion in Cardiology, vol. 15, no. 3, pp. 166-171, 2000.

[6] A. S. Curtis, Z. J. Wu, R. L. Kormos, B. P. Griffith, and J. F. Antaki, "Novel ventricular apical cannula: in vitro evaluation using transparent, compliant ventricular casts," ASAIO Journal, vol. 44, no. 5, pp. M691-M695, 1998.

[7] T. Komoda, Y. Weng, and R. Hetzer, “Technique for insertion of the inflow cannula of the INCOR left ventricular assist device," Annals of Thoracic Surgery, vol. 85, no. 4, pp. 14661467, 2008.

[8] A. Tschirkov, D. Nikolov, and V. Papantchev, "New technique for implantation of the inflow canula of Berlin Heart INCOR system," European Journal of Cardio-Thoracic Surgery, vol. 30, no. 4, pp. 678-679, 2006.

[9] G. P. Noon, D. L. Morley, S. Irwin, S. V. Abdelsayed, R. J. Benkowski, and B. E. Lynch, "Clinical experience with the MicroMed DeBakey ventricular assist device," Annals of Thoracic Surgery, vol. 71, no. 3, pp. S133-S138, 2001.

[10] G. M. Wieselthaler, H. Schima, and E. Wolner, "Special considerations on the implantation technique for the MicroMedDeBakey ventricular assist device axial pump," Annals of Thoracic Surgery, vol. 76, no. 6, pp. 2109-2111, 2003.

[11] T. Akimoto, K. Yamazaki, P. Litwak et al., "Continuously maintaining positive flow avoids endocardial suction of a rotary blood pump with left ventricular bypass," Artificial Organs, vol. 24, no. 8, pp. 606-610, 2000.

[12] C. Schmid, M. Jurmann, D. Birnbaum et al., "Influence of inflow cannula length in axial-flow pumps on neurologic adverse event rate: results from a multi-center analysis," Journal of Heart and Lung Transplantation, vol. 27, no. 3, pp. 253260, 2008.

[13] D. Lacasse, A. Garon, and D. Pelletier, "Mechanical hemolysis in blood flow: user-independent predictions with the solution of a partial differential equation.", Computer Methods in Biomechanics and Biomedical Engineering, vol. 10, no. 1, pp. 1-12, 2007.

[14] X. Song, A. L. Throckmorton, H. G. Wood, J. F. Antaki, and D. B. Olsen, "Computational fluid dynamics prediction of blood damage in a centrifugal pump," Artificial Organs, vol. 27, no. 10, pp. 938-941, 2003.

[15] A. Arvand, M. Hormes, and H. Reul, "A validated computational fluid dynamics model to estimate hemolysis in a rotary blood pump," Artificial Organs, vol. 29, no. 7, pp. 531-540, 2005.

[16] T. N. Bachman, J. K. Bhama, J. Verkaik, S. Vandenberghe, R. L. Kormos, and J. F. Antaki, "In vitro evaluation of ventricular cannulation for rotodynamic cardiac assist devices," Cardiovascular Engineering and Technology, vol. 2, no. 3, pp. 203-211, 2011.

[17] J. K. Bhama, T. N. Bachman, R. L. Kormos, H. Borovetz, and J. F. Antaki, "Development of an ex vivo ovine ventricular assist device model for intraventricular visualization of the inflow cannula," Journal of Heart and Lung Transplantation, vol. 28, no. 8, pp. 860-861, 2009.

[18] Y. Ootaki, D. Saeed, C. Ootaki et al., "Development of the DexAide right ventricular assist device inflow cannula," ASAIO Journal, vol. 54, no. 1, pp. 31-36, 2008.

[19] K. H. Fraser, T. Zhang, M. E. Taskin, B. P. Griffith, and Z. J. Wu, "Computational fluid dynamics analysis of thrombosis potential in left ventricular assist device drainage cannulae," ASAIO Journal, vol. 56, no. 3, pp. 157-163, 2010.

[20] M. Grigioni, C. Daniele, U. Morbiducci et al., "Computational model of the fluid dynamics of a cannula inserted in a vessel: incidence of the presence of side holes in blood flow," Journal of Biomechanics, vol. 35, no. 12, pp. 1599-1612, 2002.

[21] D. V. Amin, J. F. Antaki, P. Litwak, D. Thomas, Z. J. Wu, and M. Watach, "Induction of ventricular collapse by an axial flow blood pump," ASAIO Journal, vol. 44, no. 5, pp. M685-M690, 1998.

[22] T. Tsukiya, K. Toda, and H. Sumikura, "Computational fluid dynamic analysis of the flow field in the newly developed inflow cannula for a bridge-to-decision mechanical circulatory support," Artificial Organs, vol. 14, no. 4, pp. 381-384, 2011.

[23] H. Zhan-Zhong, W. Jing, and L. Xiao-Ping, FLUENT Fluid Engineering Simulation Example and Apply, Beijing Institute of Technology Press, 2007.

[24] M. Giersiepen, L. J. Wurzinger, R. Opitz, and H. Reul, "Estimation of shear stress-related blood damage in heart valve prostheses-in vitro comparison of 25 aortic valves," International Journal of Artificial Organs, vol. 13, no. 5, pp. 300-306, 1990.

[25] R. Cheng, Y. G. Lai, and K. B. Chandran, “Three-dimensional fluid-structure interaction simulation of bileaflet mechanical heart valve flow dynamics," Annals of Biomedical Engineering, vol. 32, no. 11, pp. 1471-1483, 2004.

[26] L. Goubergrits and K. Affeld, "Numerical estimation of blood damage in artificial organs," Artificial Organs, vol. 28, no. 5, pp. 499-507, 2004. 
[27] J. Wu, B. E. Paden, H. S. Borovetz, and J. F. Antaki, “Computational fluid dynamics analysis of blade tip clearances on hemodynamic performance and blood damage in a centrifugal ventricular assist device," Artificial Organs, vol. 34, no. 5, pp. 402-411, 2010.

[28] G. Song, L. P. Chua, and T. M. Lim, "Numerical study of a biocentrifugal blood pump with straight impeller blade profiles," Artificial Organs, vol. 34, no. 2, pp. 98-104, 2010. 

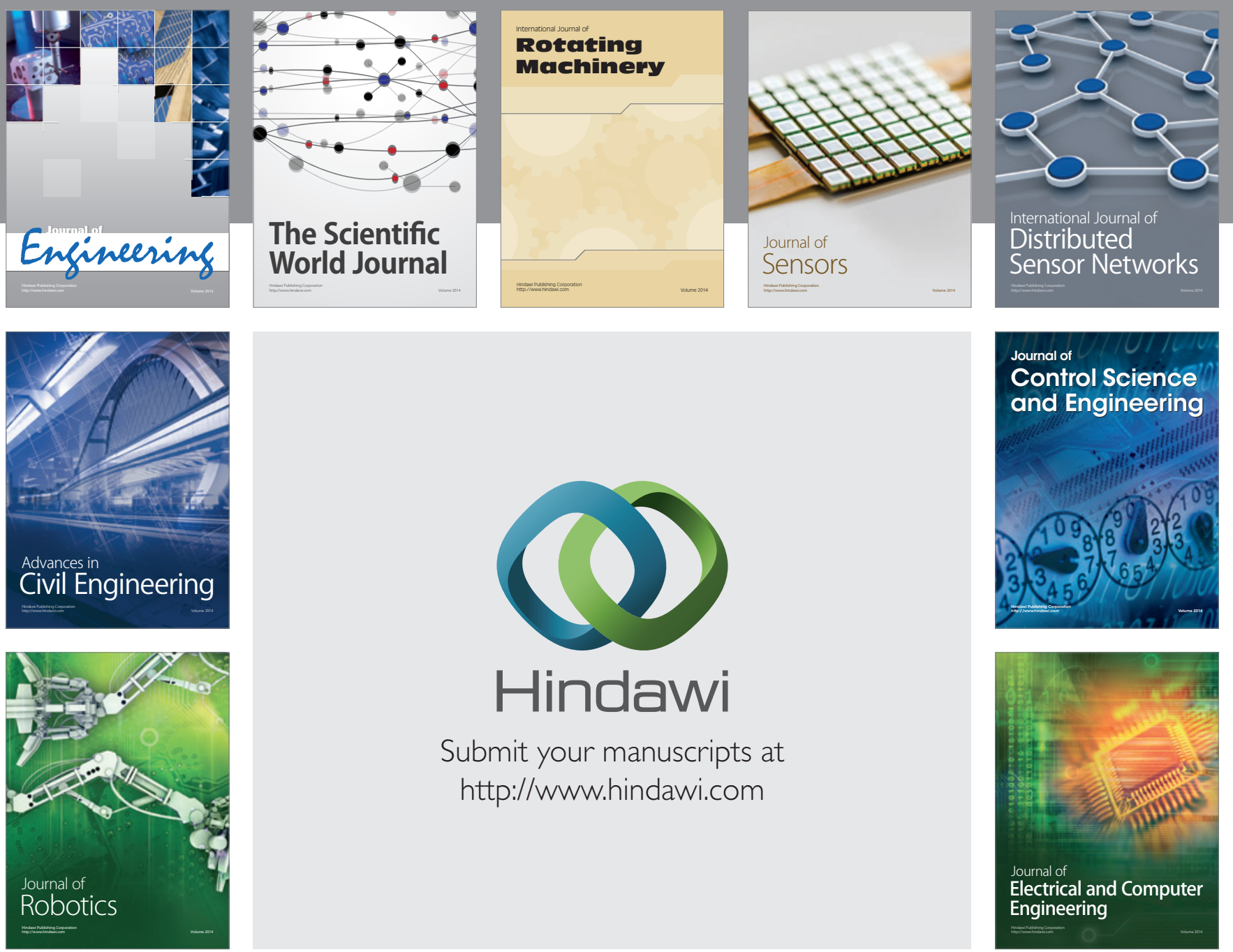

Submit your manuscripts at

http://www.hindawi.com
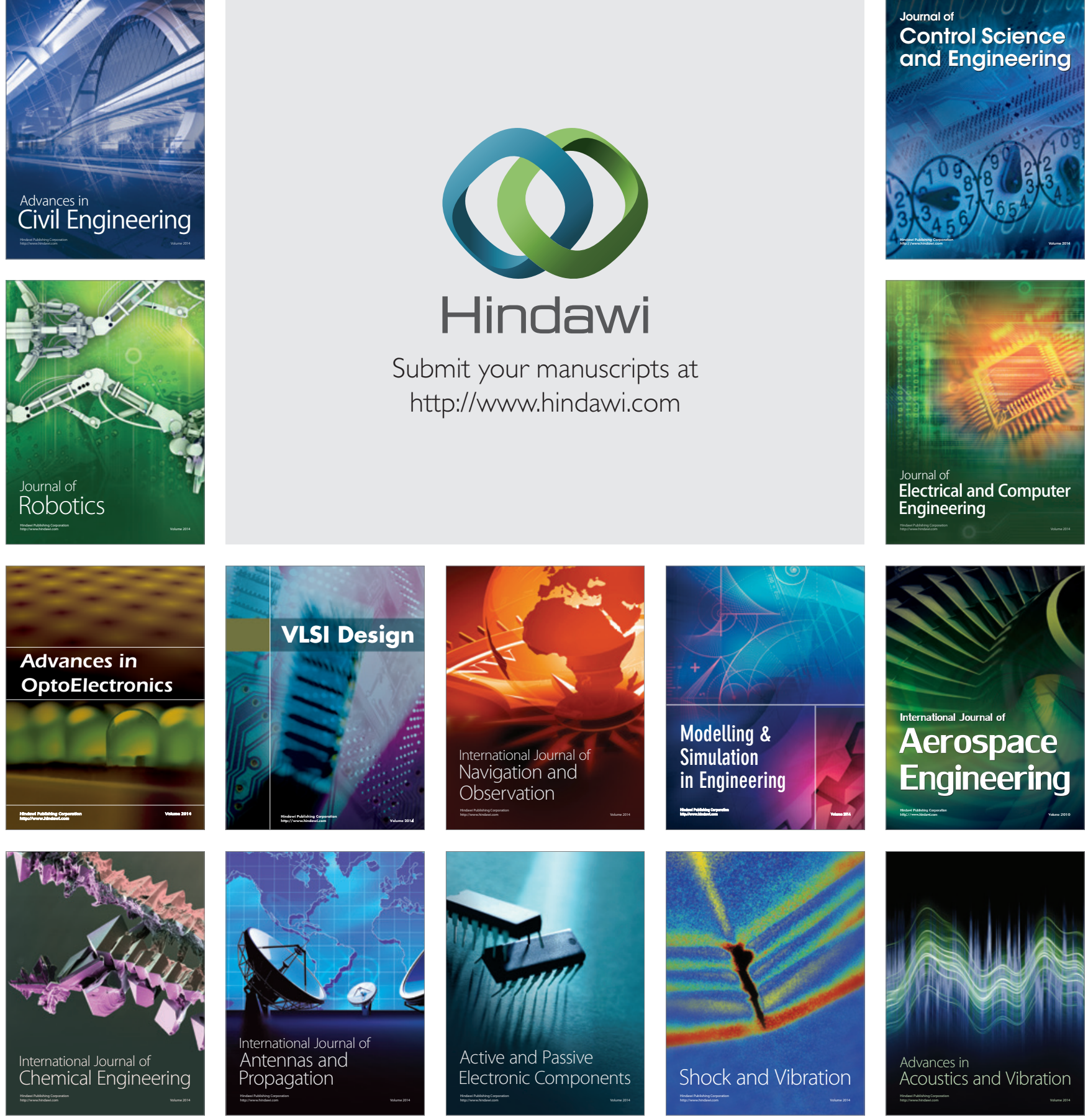